\title{
Efeitos Adversos do Sufentanil Associado ao Anestésico Local pelas Vias Subaracnóidea e Peridural em Pacientes Submetidas à Analgesia de Parto*
}

\section{Side Effects of Subarachnoid and Epidural Sufentanil Associated with a Local Anesthetic in Patients Undergoing Labor Analgesia}

\author{
Isabel C.F. Salem', Fernanda B. Fukushima², Giane Nakamura ${ }^{3}$, Fábio Ferrari, TSA ${ }^{3}$, Laís C. Navarro ${ }^{3}$ \\ Yara Marcondes Machado Castiglia, TSA ${ }^{4}$, Eliana Marisa Ganem, TSA
}

\section{RESUMO}

Salem ICF, Fukushima FB, Nakamura G, Ferrari F, Navarro LC, Castiglia YMM, Ganem EM - Efeitos Adversos do Sufentanil Associado ao Anestésico Local pelas Vias Subaracnóidea e Peridural em Pacientes Submetidas à Analgesia de Parto.

JUSTIFICATIVA E OBJETIVOS: A associação do opióide ao anestésico local melhora a qualidade da analgesia de parto e reduz o risco de toxicidade sistêmica pelo anestésico local. Os opióides, entretanto, podem determinar efeitos colaterais. O objetivo desta pesquisa foi comparar os efeitos adversos determinados pelo sufentanil, administrado por via subaracnóidea, associado à bupivacaína, com aquele determinado pelo sufentanil por via peridural, associado à ropivacaína, nas doses utilizadas no Serviço de Anestesia, em gestantes submetidas à analgesia de parto.

MÉTODO: Participaram do estudo 60 pacientes, estado físico ASA I e II, com idade entre 15 e 42 anos, com gestação a termo e fetos saudáveis, submetidas à analgesia de parto. Foram distribuídas de forma aleatória em dois grupos: G1 - Duplo bloqueio - bupivacaína a 0,5\% (2,5 mg) e sufentanil $(5 \mu \mathrm{g})$ pela via subaracnóidea, G2 - Peridural - ropivacaína a 0,2\% (20 mg) e sufentanil $(10 \mu \mathrm{g})$ pela via peridural. Para doses complementares foi administrada ropivacaína a 0,2\% (12 $\mathrm{mg})$ e para resolução do parto, ropivacaína a 1\% (50 mg). As pacientes foram avaliadas após analgesia (M1) com relação a hipotensão arterial, bradicardia materna, prurido, náusea, vômito, depressão respiratória e sedação. No pós-operatório (M2), quanto à presença de náusea, vômito, prurido, sedação, retenção urinária e dor. Os recém-nascidos foram avaliados pelo índice de Apgar. Para análise estatística, foram utilizados teste $t$ de Student, Mann-Whitney e Qui-quadrado.

RESULTADOS: Os grupos foram similares com relação à idade, ao peso, à altura, à duração do período de trabalho de parto após analgesia, ao Apgar dos recém-nascidos, à ocorrência de hipotensão arterial, bradicardia, náusea, vômito, prurido e retenção urinária. A sedação foi mais freqüente nas pacientes de G2, em M1 (50\%) com diferença estatística significativa.

\footnotetext{
${ }^{*}$ Recebido do (Received from) CET/SBA da Faculdade de Medicina de Botucatu, Universidade Estadual de São Paulo (FMB - UNESP), Botucatu, SP

1. Graduanda do $5^{\circ}$ Ano de Medicina da FMB - UNESP; Bolsa de Iniciação Científica FAPESP

2. $M E_{3}$ (2003) do CET/SBA da FMB - UNESP

3. Médico do CET/SBA do Departamento de Anestesiologia da FMB - UNESP

4. Professora Titular do CET/SBA do Departamento de Anestesiologia da FMB - UNESP

5. Professora Adjunta do CET/SBA do Departamento de Anestesiologia da FMB - UNESP

Apresentado (Submitted) em 03 de abril de 2006

Aceito (Accepted) para publicação em 05 de dezembro de 2006

Endereço para correspondência (Correspondence to):

Profa. Dra. Eliana Marisa Ganem

Depto. de Anestesiologia da FMB - UNESP

18618-970 Botucatu, SP

E-mail: eganem@fmb.unesp.br

(C) Sociedade Brasileira de Anestesiologia, 2007
}

CONCLUSÕES: O sufentanil nas doses utilizadas, administrado por via subaracnóidea ou peridural, associado aos anestésicos locais, determinou similaridade na duração do trabalho de parto após analgesia e no Apgar dos recém-nascidos. A sedação foi o efeito adverso mais freqüente nas pacientes que receberam o opióide pela via peridural.

Unitermos: ANALGESIA, Obstétrica: parto; ANALGÉSICOS, Opióides: sufentanil; ANESTÉSICOS, Local: bupivacaína, ropivacaína; COMPLICAÇÕES: bradicardia, hipotensão arterial, náusea, prurido, vômito, sedação; TÉCNICAS ANESTÉSICAS, Regional: subaracnóidea peridural combinada, peridural.

\section{SUMMARY}

Salem ICF, Fukushima FB, Nakamura G, Ferrari F, Navarro LC, Castiglia YMM, Ganem EM - Side Effects of Subarachnoid and Epidural Sufentanil Associated with a Local Anesthetic in Patients Undergoing Labor Analgesia.

BACKGROUND AND OBJECTIVES: The association of an opioid with a local anesthetic improves the quality of labor analgesia and reduces the risk of systemic toxicity of the local anesthetic. However, opioids are not devoid of side effects. The aim of this study was to compare the side effects of subarachnoid sufentanil associated with bupivacaine to those caused by epidural sufentanil associated with ropivacaine in the doses used in the Anesthesiology Department in pregnant women undergoing labor analgesia.

METHODS: Sixty pregnant women, ASA physical status I and II, ages between 15 and 42 years, at term and with healthy fetuses, undergoing labor analgesia were enrolled in this study. They were randomly divided in two groups: $G 1$ - combined spinal epidural anesthesia - $0.5 \%$ bupivacaine $(2.5 \mathrm{mg})$ and subarachnoid sufentanil $(5 \mu \mathrm{g})$; $\mathrm{G} 2$ Epidural Block - $0.2 \%$ ropivacaine $(20 \mathrm{mg})$, and epidural sufentanil $(10 \mu \mathrm{g})$. Complementary doses of $0.2 \%$ ropivacaine $(12 \mathrm{mg})$ were administered whenever necessary, and $1 \%$ ropivacaine $(50 \mathrm{mg})$ was administered for labor resolution. Patients were evaluated after analgesia (M1) regarding the presence of hypotension, maternal bradycardia, pruritus, nausea, vomiting, respiratory depression, and sedation. They were also evaluated postoperatively (M2) regarding the presence of nausea, vomiting, pruritus, sedation, urinary retention, and pain. Newborns were evaluated by the Apgar score. The test $t$ Student, MannWhitney test, and Chi-Square test were used for the statistical analysis. RESULTS: Both groups were similar regarding age, weight, height, duration of labor after analgesia, Apgar score of the newborns, hypotension, maternal bradycardia, nausea, vomiting, pruritus, and urinary retention. Sedation was more frequent in patients in G2 at M1 (50\%), which was statistically significant.

CONCLUSION: Subarachnoid or epidural sufentanil, in the doses used in this study, associated with local anesthetics, had the same effect on the duration of labor after analgesia and in the Apgar score of newborns. Sedation was the most frequent side effect in patients receiving epidural sufentanil.

Key Words: ANALGESIA, Obstetric: labor; ANALGESICS, Opioids: sufentanil; ANESTHETICS, Local: bupivacaine, ropivacaine; ANESTHETIC TECHNIQUES, Regional: combined subarachnoid and epidural, epidural; COMPLICATIONS: bradycardia, hypotension, nausea, pruritus, vomiting, sedation. 


\section{INTRODUÇÃO}

A dor representa um dos mais importantes sinais de início do trabalho de parto e, estabelecido o seu diagnóstico e a regularidade das contrações uterinas, ela deve ser aliviada, visto que apresenta uma série de efeitos indesejáveis para a mãe e o feto.

A ansiedade e a dor durante a fase de dilatação determinam hiperventilação materna, aumento no consumo de oxigênio e nas concentrações de catecolaminas circulantes, cortisol e hormônio adrenocorticotrófico ${ }^{1}$. A hiperventilação ocasiona hipocarbia, vasoconstrição útero-placentária e desvio da curva de dissociação da hemoglobina materna para a esquerda, efeitos que, associados ao aumento do consumo de oxigênio materno, diminuem a oferta de oxigênio ao feto. Concentrações excessivas de catecolaminas circulantes, além de vasoconstrição uterina ${ }^{1}$, aumentam o consumo de oxigênio ${ }^{1}$ e o lactato sangüíneo ${ }^{1}$. O alívio da dor durante o trabalho de parto diminui a ventilação-minuto, o consumo de oxigênio, as concentrações de catecolaminas circulantes e de lactato sangüíneo ${ }^{1}$. Estudos realizados após anestesia peridural para analgesia de parto mostraram que houve melhora na perfusão placentária e redução na concentração de substâncias liberadas durante o estresse, como cortisol e $\beta$-endorfinas ${ }^{1}$.

$\mathrm{O}$ advento de técnicas anestésicas modernas, o desenvolvimento de novos anestésicos locais com menor toxicidade cardiovascular e neurológica e a adição de opióides ao anestésico local proporcionam parto livre de dor com mínimos efeitos colaterais maternos e fetais ${ }^{1-3}$.

$\mathrm{O}$ anestésico local $(\mathrm{AL})$ determina bloqueio dos canais de sódio da membrana dos axônios das raízes nervosas espinhais e das células do corno posterior e anterior da medula. Os opióides, por sua vez, atuam como agonistas nos receptores opióides em locais pré- e pós-sinápticos do sistema nervoso central e em outras áreas do organismo ${ }^{4,5}$. Com mecanismos de ação distintos, esses fármacos associados têm ação sinérgica ${ }^{6}$.

A adição de opióides, como fentanil e sufentanil, ao AL diminui a latência, prolonga a duração e melhora a qualidade da anestesia peridural ${ }^{1}$. Essa associação também permite a utilização de baixas concentrações de $A L$, o que resulta em menor bloqueio motor e menor risco de toxicidade ${ }^{1}$.

Outra técnica muito utilizada para alívio da dor durante o trabalho de parto é a de duplo bloqueio (anestesia subaracnóidea e peridural), na qual se preconiza a associação de bupivacaína aos opióides lipossolúveis (fentanil ou sufentanil) pela via subaracnóidea ${ }^{7}$. As vantagens da administração subaracnóidea de opióides incluem melhora na qualidade da analgesia ${ }^{8}$, rápido início de ação ${ }^{8}$, ausência de bloqueio motor ${ }^{8}$, baixa exposição materno/fetal a fármacos e facilidade de realização da técnica ${ }^{9}$. A principal desvantagem da utilização do opióide no neuroeixo seria o aparecimento de prurido, hipotensão arterial, alterações temporárias da freqüência cardíaca fetal e depressão res- piratória, cuja incidência varia de 0,01\% a 0,1\% e não é significativa na ausência de sedação ${ }^{10}$.

Reduzindo-se a dose do opióide administrada pela via subaracnóidea, diminui-se a incidência de efeitos colaterais ${ }^{11}$. Quando se utilizou o sufentanil na dose de $5 \mu \mathrm{g}$ associado à bupivacaína pela via subaracnóidea, na técnica de duplo bloqueio, foi preservada a qualidade da analgesia com mínimos efeitos colaterais ${ }^{12}$.

O objetivo desta pesquisa foi comparar os efeitos adversos determinados pelo sufentanil, administrado pela via subaracnóidea, associado à bupivacaína, com aquele determinado pelo sufentanil por via peridural, associado à ropivacaína, nas doses utilizadas no Serviço de Anestesia, em gestantes submetidas à analgesia de parto.

\section{MÉTODO}

Após a aprovação do Comitê de Ética em Pesquisa Clínica e o consentimento por escrito, participaram do estudo 60 gestantes, com idade entre 15 e 42 anos, estado físico ASA I e II, com gestação a termo e fetos saudáveis e sem sofrimento, que foram submetidas à analgesia do trabalho de parto. Foram excluídas do estudo aquelas com queixas gástricas atuais ou prévias, as usuárias de álcool e drogas, as que apresentavam doenças psiquiátricas e as pacientes que receberam meperidina durante o trabalho de parto. As gestantes foram distribuídas, aleatoriamente, em dois grupos de estudo que se diferenciaram pela técnica anestésica utilizada, ou seja:

- Grupo 1 (duplo bloqueio): bupivacaína a 0,5\% (2,5 mg) + sufentanil $(5 \mu \mathrm{g})$ por via subaracnóidea

- Grupo 2 (peridural): ropivacaína a 0,2\% (14 mg) + sufentanil $(10 \mu \mathrm{g})$ por via peridural

Doses complementares: ropivacaína a 0,2\% (12 mg) por via peridural em ambos os grupos. Dose para resolução do parto: ropivacaína a $1 \%(50 \mathrm{mg})$ por via peridural.

Após a indicação de analgesia de parto, que no Hospital de Clínicas de Botucatu ocorre com dilatação cervical de $6 \mathrm{~cm}$, foi instalada infusão de solução de Ringer com lactato (8 a $10 \mathrm{~mL} . \mathrm{kg}^{-1}$ ) e realizada a monitorização com eletrocardioscópio na derivação $D_{\|}$, esfigmomanômetro e oxímetro de pulso.

A seguir, nas pacientes do Grupo 1, realizou-se a punção peridural na posição sentada, pela via mediana, no espaço $\mathrm{L}_{3}-\mathrm{L}_{4}$ com agulha Touhy $80 / 18$, e a inserção do cateter peridural e, em seguida, a punção subaracnóidea, no espaço $L_{2}-L_{3}$, com agulha Quincke $25 \mathrm{G}$, seguida da administração de $5 \mu \mathrm{g}$ de sufentanil e $2,5 \mathrm{mg}$ de bupivacaína. Nos casos em que a paciente necessitou de nova dose de analgesia, administrou-se, pelo cateter, dose-teste de anestésico local (lidocaína a 1\% - $30 \mathrm{mg}$ e adrenalina 1:200.000) para se assegurar quanto ao seu correto posicionamento. Após esse conhecimento, foram administrados $6 \mathrm{~mL}$ (12 $\mathrm{mg}$ ) de ropivacaína a $0,2 \%$. 
Nas pacientes do Grupo 2, a punção peridural foi realizada com a mesma técnica utilizada no Grupo 1 e, após a doseteste, foram administrados ropivacaína, a 0,2\%, em volume de $10 \mathrm{~mL}(20 \mathrm{mg})$, e sufentanil $(10 \mu \mathrm{g})$. Quando as pacientes necessitaram de doses adicionais de anestésicos, porque apresentavam dor, foi administrada dose complementar de ropivacaína a $0,2 \%$ em volume $6 \mathrm{~mL}(12 \mathrm{mg})$.

Para resolução do parto, foram administrados $5 \mathrm{~mL}$ de ropivacaína a $1 \%(50 \mathrm{mg})$.

A pesquisa dos efeitos adversos foi efetuada em dois momentos: M1 - período de trabalho de parto após analgesia, M2 - 24 horas após a realização da analgesia.

O intervalo de tempo que se iniciou com a realização da analgesia e terminou com a saída da paciente da sala de parto foi considerado como período de trabalho de parto após a analgesia, no qual foi pesquisada, a cada 10 minutos, a presença de hipotensão arterial (diminuição de pelo menos $20 \%$ dos valores iniciais da pressão arterial sistólica), bradicardia (freqüência cardíaca menor que 60 batimentos por minuto), prurido, sedação, náusea, vômito e depressão respiratória. Os recém-nascidos foram avaliados pelo boletim de Apgar.

Caso ocorresse hipotensão arterial, ela deveria ser inicialmente tratada com deslocamento do útero para a esquerda e aumento da infusão de líquidos. Se essas manobras fossem ineficientes seria administrado cloridrato de efedrina. Para o tratamento do prurido seria utilizado o propofol (10 $\mathrm{mg}$ ), e da náusea, o ondansetron (4 mg).

Nas primeiras 24 horas após a realização da analgesia, as parturientes foram orientadas a solicitar a presença de um anestesiologista se apresentassem prurido, náusea e dor quando recebessem a medicação preestabelecida em protocolo para tratamento do efeito adverso.

No pós-operatório, 24 horas após a realização da analgesia (M2), as pacientes receberam a visita de outro anestesiologista que tinha participado da anestesia e que pesquisava a presença de dor, náusea, vômito, prurido, sedação e retenção urinária. Esses eventos foram avaliados por meio de entrevista, sem que se soubesse a qual grupo de estudo pertencia a parturiente.

A sedação foi avaliada pela escala numérica de sedação que variou de 0 a 3 , sendo $0=$ paciente acordada, sem evidência clínica de sedação; 1 = sonolenta, respondendo ao comando verbal; 2 = sonolenta não respondendo ao comando verbal, mas respondendo ao toque leve; e $3=\mathrm{com}$ sedação intensa, respondendo ao estímulo doloroso. O prurido também foi avaliado por meio de escala numérica que variou de 0 a 3 : sendo $0=$ prurido ausente; 1 = prurido presente, leve, sem necessidade de coçar; 2 = prurido moderado, sendo necessário coçar ocasionalmente; e $3=$ prurido intenso, coceira constante. A dor foi avaliada pela escala numérica verbal que variou de 0 a 10 , sendo 0 a ausência de dor e 10 a dor máxima. Quando a intensidade da dor superou 3 , foi administrado cetoprofeno $(50 \mathrm{mg}$ ) por via oral, conforme conduta estabelecida pelo Serviço de Obstetrícia deste hospital.

Considerando-se a diferença entre os grupos de $40 \%$ e poder do teste de $95 \%$, o tamanho da amostra ficou determinado em, no mínimo, 22 pacientes por grupo.

Foi realizada análise estatística dos resultados, para variáveis quantitativas com distribuição normal foi realizado o teste $t$ de Student, caso contrário o teste de Mann-Whitney. Para variáveis qualitativas foi utilizado o teste do Qui-quadrado.

\section{RESULTADOS}

Os grupos foram homogêneos com relação aos dados antropométricos, à duração do período do trabalho de parto após analgesia (Tabela I) e ao boletim de Apgar dos recém-nascidos (Tabela II).

Apenas duas pacientes pertencentes ao Grupo 1 necessitaram de dose complementar de anestésico local por via peridural. Em ambos os casos foram doses únicas administradas após 95 e 110 minutos da dose inicialmente introduzida no espaço subaracnóideo. Cinco pacientes do Grupo 2 necessitaram de doses complementares de ropivacaína, também em doses únicas em intervalo de tempo que variou de 100 a 195 minutos após a dose inicial.

Das 60 pacientes selecionadas para participar do estudo, oito foram excluídas da análise dos resultados porque foram submetidas à cesariana. Dentre as 52 restantes, 28 pertenciam ao Grupo 1 e 24 ao Grupo 2.

Cinco pacientes do Grupo 1 e quatro do Grupo 2 foram submetidas à episiotomia para realização de fórcipe de alívio.

Dentre as 28 gestantes pertencentes ao Grupo 1, 19 apresentaram um ou mais eventos adversos durante o período de estudo; destas, três no M1, oito no M1 e no M2 e oito apenas no $M 2$. O sintoma mais comum no M1 foi o prurido, quei-

Tabela I - Dados Antropométricos e Tempo de Duração do M1 (Média \pm DP)

\begin{tabular}{lccc}
\hline & Grupo 1 & Grupo 2 & Estatística \\
\hline Idade (anos) & $20,6 \pm 4,1$ & $22,6 \pm 6,6$ & $\mathrm{G} 1=\mathrm{G} 2$ \\
Peso $(\mathrm{kg})$ & $72,4 \pm 8,2$ & $72,1 \pm 10,4$ & $\mathrm{G} 1=\mathrm{G} 2$ \\
Altura $(\mathrm{cm})$ & $162 \pm 6$ & $161 \pm 6$ & $\mathrm{G} 1=\mathrm{G} 2$ \\
Duração do M1 (min) & $152 \pm 3$ & $175 \pm 14$ & $\mathrm{G} 1=\mathrm{G} 2$ \\
\hline
\end{tabular}

Tabela II - Boletim de Apgar dos Recém-Nascidos. Resultados Expressos em Mediana

\begin{tabular}{lccc}
\hline Momento & Grupo 1 & Grupo 2 & Estatística \\
\hline $1 \min$ & $8[7,5-8,5]$ & $8[8-9]$ & $\mathrm{G} 1=\mathrm{G} 2$ \\
$5 \mathrm{~min}$ & $9[9-10]$ & $9[9-10]$ & $\mathrm{G} 1=\mathrm{G} 2$ \\
$10 \mathrm{~min}$ & $10[9-10]$ & $10[9-10]$ & $\mathrm{G} 1=\mathrm{G} 2$ \\
\hline
\end{tabular}


xa de oito pacientes (seis de leve e duas de moderada intensidade), seguido de hipotensão arterial e náusea, observadas em quatro gestantes (Tabela III). As pacientes que apresentaram diminuição na pressão arterial não foram as mesmas que se queixaram de náusea. O prurido não necessitou de tratamento. A hipotensão arterial foi tratada com deslocamento uterino para a esquerda e aumento da infusão de líquido. A náusea foi temporária e não necessitou de tratamento. No período $\mathrm{M} 2$, prurido foi a queixa de oito pacientes, seguido de dor e sedação, observadas em seis gestantes (Tabela IV).

No Grupo 2, 18 pacientes apresentaram um ou mais efeitos adversos durante o período de avaliação, oito apenas no $M 1$, sete no $M 1$ e no $M 2$ e três apenas no M2. A sedação foi a queixa de 12 pacientes no M1 (Tabela III) e de cinco no M2, e quatro destas já haviam referido sedação no M1. Dez gestantes apresentaram sedação de grau 1 e três de grau 2. 0 prurido foi o segundo efeito adverso mais observado, classificado de intensidades leve e moderada (duas no M1 e quatro pacientes no M2) (Tabela IV), não necessitando de tratamento. A ocorrência de sedação nas pacientes do Grupo 2, no M1, foi maior que no Grupo 1, com diferença estatística significativa ( $p=0,017)$.

Tabela III - Efeitos Adversos Observados no M1

\begin{tabular}{lccc}
\hline & $\begin{array}{c}\text { Grupo 1 } \\
\mathrm{n}(\%)\end{array}$ & $\begin{array}{c}\text { Grupo } 2 \\
\mathrm{n}(\%)\end{array}$ & Estatística \\
\hline Hipotensão arterial & $4(14)$ & $1(4)$ & $\mathrm{G} 1=\mathrm{G} 2$ \\
Bradicardia & 0 & $1(4)$ & $\mathrm{G} 1=\mathrm{G} 2$ \\
Prurido & $8(29)$ & $2(8)$ & $\mathrm{G} 1=\mathrm{G} 2$ \\
Náusea & $4(14)$ & 0 & $\mathrm{G} 1=\mathrm{G} 2$ \\
Vômito & $2(7)$ & 0 & $\mathrm{G} 1=\mathrm{G} 2$ \\
Depressão respiratória & 0 & 0 & $\mathrm{G} 1=\mathrm{G} 2$ \\
Sedação & $2(7)$ & $12(50)$ & $\mathrm{G} 1<\mathrm{G} 2$ * \\
\hline
\end{tabular}

${ }^{*} p<0,05$

Tabela IV - Efeitos Adversos Observados no M2. Resultados Expressos em Número Absoluto (n) e Porcentagem (\%)

\begin{tabular}{lccc}
\hline & $\begin{array}{c}\text { Grupo } 1 \\
\mathrm{n}(\%)\end{array}$ & $\begin{array}{c}\text { Grupo } 2 \\
\mathrm{n}(\%)\end{array}$ & Estatística \\
\hline Dor & $7(25)$ & $3(12,5)$ & $\mathrm{G} 1=\mathrm{G} 2$ \\
Náusea & $3(11)$ & $3(12,5)$ & $\mathrm{G} 1=\mathrm{G} 2$ \\
Vômito & 0 & 0 & $\mathrm{G} 1=\mathrm{G} 2$ \\
Prurido & $9(32)$ & $4(17)$ & $\mathrm{G} 1=\mathrm{G} 2$ \\
Sedação & $6(21)$ & $5(21)$ & $\mathrm{G} 1=\mathrm{G} 2$ \\
Retenção urinária & $4(14)$ & $1(4)$ & $\mathrm{G} 1=\mathrm{G} 2$ \\
\hline
\end{tabular}

\section{DISCUSSÃO}

Os resultados mostraram que o sufentanil nas doses utilizadas neste estudo, administrado por via subaracnóidea ou peridural e associado aos anestésicos locais, apresentou comportamento similar quanto à duração da analgesia e à vitalidade dos recém-nascidos, avaliadas pelo boletim de Apgar.

A ocorrência de efeitos adversos, como prurido, bradicardia, hipotensão arterial, náusea, vômito, depressão respiratória e retenção urinária também foi igual, em ambos os grupos durante o período de estudo. Apenas a incidência de sedação em M1 foi maior nas gestantes que receberam sufentanil por via peridural, valores com diferença estatística significativa.

A administração de sufentanil no neuroeixo está associada a muitos efeitos colaterais. Embora alguns desses efeitos reflitam a sua ação espinhal, outros são causados por migração cefálica e efeitos supra-espinhais ${ }^{13}$.

O prurido é causado por ações espinhais e supra-espinhais ${ }^{11}$. É o efeito adverso mais freqüente ${ }^{14}$. Apesar de não ter ocorrido diferença estatística significativa, o prurido foi queixa de $32 \%$ das pacientes que foram submetidas à técnica de duplo-bloqueio e de $17 \%$ das submetidas à anestesia peridural.

Esses resultados estão de acordo com os descritos na literatura, nos quais o relato de prurido é maior após a administração de opióide pela via subaracnóidea ${ }^{15}$. A incidência, após injeção subaracnóidea de sufentanil, é elevada, variando de $25 \%$ a $90 \%{ }^{11-13,16-18}$.

Em estudo com parturientes submetidas à analgesia de parto com a técnica de duplo-bloqueio, nas quais foi administrado o sufentanil, nas doses de $5 \mu \mathrm{g}$ ou $10 \mu \mathrm{g}$, foi observado que a redução na dose do opióide não alterou a freqüência ou a intensidade do prurido ${ }^{13}$.

Alguns autores ${ }^{11}$ observaram que $5 \mu \mathrm{g}$ de sufentanil, introduzidos no espaço subaracnóideo, ocasionaram menor incidência de hipotensão arterial e sedação que os $10 \mu \mathrm{g}$, porém a ocorrência de prurido não variou com a redução da dose. Resultados idênticos foram observados com doses de sufentanil que variaram de $2,5 \mu \mathrm{g}$ a $10 \mu \mathrm{g}{ }^{18}$.

De maneira similar ao que ocorre com os efeitos analgésicos, parece haver um limiar acima do qual o sufentanil produz prurido independentemente da dose administrada ${ }^{13}$. Ainda não está esclarecido se o prurido é induzido pela migração cefálica do fármaco no líquor e subseqüente interação com o núcleo do nervo trigêmeo, localizado na medula ${ }^{19}$, ou se há envolvimento com os receptores da serotonina do tipo 3, que são abundantes no corno dorsal da medula espinhal ${ }^{19}$.

Já $14 \%$ das pacientes que foram submetidas à técnica de duplo-bloqueio foram acometidas por hipotensão arterial, resultados que são similares aos descritos por outros autores ${ }^{7,11,12}$. 
A hipotensão arterial, desencadeada pela injeção subaracnóidea e peridural de sufentanil, é dependente da dose do opióide administrada, assim como da associação ao anestésico local e à dose utilizada.

Em diversos estudos, em gestantes, ficou demonstrado que associação de bupivacaína $(2,5 \mathrm{mg})$ com sufentanil $(10 \mu \mathrm{g})$, pela via subaracnóidea, determinou hipotensão arterial com freqüência que variou de $11 \%^{7}$ a $43 \%{ }^{11}$. A dose de 7,5 $\mu \mathrm{g}$, adicionada à bupivacaína $(2,0 \mathrm{mg})$, causou redução na pressão arterial em 15\% das parturientes e a dose de $5 \mu \mathrm{g}$ com bupivacaína $(1,25 \mathrm{mg})$ determinou incidência entre $5 \%$ e $9 \%{ }^{11,12}$.

Utilizando método não-invasivo de bioimpedância torácica para realização de monitorização hemodinâmica central, em parturientes submetidas à técnica de duplo-bloqueio nas quais o sufentanil $(10 \mu \mathrm{g})$ foi administrado no espaço subaracnóideo, foi observada hipotensão arterial em 10\% que não foi acompanhada de alterações nos parâmetros cardiovasculares centrais ${ }^{20}$.

Está estabelecido que a hipotensão arterial secundária à administração subaracnóidea de sufentanil, em pacientes submetidas à analgesia de parto, é decorrente da rápida e profunda analgesia determinada pela técnica anestésica, o que leva à diminuição imediata das concentrações de catecolaminas circulantes ${ }^{21}$.

Quanto à náusea, foi a queixa de $14 \%$ das gestantes submetidas ao duplo-bloqueio em $\mathrm{M} 1$, e de $12 \%$ em ambos os grupos em $\mathrm{M} 2$.

A incidência de náusea em gestantes após administração subaracnóidea de sufentanil com doses entre $1 \mu \mathrm{g}$ e $10 \mu \mathrm{g}$ variou de $0 \%$ a $35 \%^{7,11,12,18,21}$.

Quando o sufentanil $(10 \mu \mathrm{g})$ foi adicionado à ropivacaína a $0,2 \%$ e administrado no espaço peridural para analgesia de parto, $20 \%$ das gestantes apresentaram náusea ${ }^{23}$.

Em analgesia pós-operatória de intervenções cirúrgicas abdominais, a infusão contínua de sufentanil pela via peridural determinou êmese em $33 \%$ dos pacientes ${ }^{24}$.

Associado à bupivacaína para tratamento de dor após cesariana, o sufentanil causou náusea em $12 \%$ das pacientes ${ }^{25}$.

Alguns autores, entretanto, não constataram êmese em pacientes que receberam até $30 \mu \mathrm{g}$ de sufentanil pela via peridural para tratamento de dor pós-operatória de cesariana ${ }^{25}$.

Os opióides podem desencadear náusea e vômito porque estimulam a área quimiorreceptora de gatilho no assoalho do quarto ventrículo, refletindo sua característica de agonistas parciais dos receptores dopaminérgicos da zona quimiorreceptora. A barreira hematoencefálica é mais permeável nesse local do que em outras partes do encéfalo ${ }^{26}$

Quanto à sedação, 50\% das pacientes que receberam sufentanil por via peridural apresentaram-na. Esses resultados são superiores aos encontrados na literatura.
Vertommen e col. ${ }^{27}$ observaram que apenas $8 \%$ das pacientes submetidas à técnica de peridural com bupivacaína $(12,5 \mathrm{mg})$ e sufentanil $(10 \mu \mathrm{g})$ para analgesia do trabalho de parto apresentaram sonolência de leve intensidade.

A sonolência também foi relatada por $30 \%$ de pacientes submetidas à cesariana que receberam sufentanil $(30 \mu \mathrm{g})$ por via peridural ${ }^{28}$.

Já outros autores que administraram ropivacaína a $0,1 \%$ (10 mL) associada ao fentanil $\left(2 \mu \mathrm{g} \cdot \mathrm{mL}^{-1}\right)$ em infusão contínua para analgesia de parto constataram sonolência em $25 \%$ das gestantes ${ }^{29}$. Foi observado, entretanto, que a sedação desencadeada pelo sufentanil é mais pronunciada que aquela desencadeada pelo fentanil ${ }^{24}$.

Em analgesia pós-operatória de intervenções cirúrgicas abdominais, o sufentanil, em infusão contínua $\left(5 \mu \mathrm{g} \cdot \mathrm{h}^{-1}\right)$ pela via peridural, promoveu sedação leve e nas primeiras quatro horas de infusão ${ }^{24}$. Na presente pesquisa, a maior freqüência de sedação foi observada em M1, também tendo sido precoce nas três horas iniciais após a administração do sufentanil.

Já a ocorrência de sedação em $\mathrm{M} 1$, nas pacientes submetidas ao duplo bloqueio, foi de $7 \%$.

A incidência de sedação em pacientes obstétricas, submetidas ao duplo bloqueio e que receberam sufentanil isoladamente ou associado ao anestésico local pela via subaracnóidea, é muito variada.

É descrita sedação de leve intensidade em $100 \%$ de parturientes que receberam $10 \mu \mathrm{g}$ de sufentanil no espaço subaracnóideo ${ }^{25}$. Outro estudo obteve resultado semelhante com essa dose ${ }^{30}$.

Outros autores relataram que doses de sufentanil que variaram de $1 \mu \mathrm{g}$ a $10 \mu \mathrm{g}$ no espaço subaracnóideo, para analgesia de parto, causaram sonolência em $30 \%$ das pacientes, independentemente da dose administrada. Em outro estudo, a introdução de $8 \mu \mathrm{g}$ de sufentanil no espaço subaracnóideo originou sedação em $58 \%$ das gestantes ${ }^{31}$.

Quando associado à bupivacaína, o sufentanil $(7,5 \mu \mathrm{g})$ desencadeou sedação leve em $12,5 \%$ das gestantes ${ }^{22}$. Após $5 \mu \mathrm{g}, 5 \%$ apresentaram o sintoma ${ }^{11}$.

Embora a sedação seja efeito colateral comum dos opióides, o mecanismo e as características deste fenômeno não são totalmente conhecidos. Em estudo sobre os prováveis mecanismos que atuam na interação entre os opióides e benzodiazepínicos, há a hipótese de existência de co-localização dos receptores do ácido gama-aminobutírico (GABA) e dos receptores opióides no sistema nervoso central, com possível atividade cruzada e vias comuns de transdução intracelular ${ }^{32}$.

Para que os opióides, administrados pela via peridural, possam exercer seus efeitos, eles devem atravessar a duramáter e aracnóide, difundir-se através do líquor, atravessar a pia-máter, atingir a superfície da medula espinhal, difundir-se através da substância branca e, então, da substância cinzenta para alcançar os receptores opióides no corno dorsal ${ }^{33}$. 
No espaço peridural, os opióides podem sofrer captação pela gordura peridural, e absorção sistêmica ou difundirse através da dura-máter para o líquor ${ }^{34}$. Como o espaço peridural contém extenso plexo venoso, a absorção vascular é intensa. A administração peridural de fentanil e sufentanil produz concentrações sangüíneas similares àquelas produzidas por injeção venosa de doses equivalentes ${ }^{34}$.

Alguns autores demonstraram que o fentanil exerce efeitos espinhais, quando administrado por via peridural ${ }^{35}$, e diversos estudos mostraram que o fentanil ${ }^{36}$ e o sufentanil ${ }^{37}$, quando administrados pela via peridural, produziram a mesma intensidade de analgesia e os mesmos efeitos colaterais observados quando foram administrados por via venosa. Essas pesquisas ressaltam a importância da absorção sistêmica e subseqüente distribuição encefálica na qualidade da analgesia e intensidade dos efeitos colaterais, quando se utilizam os opióides lipofílicos ${ }^{33}$.

Em estudo prospectivo, aleatório e duplamente encoberto, utilizando infusão contínua de sufentanil $\left(0,2 \mu \mathrm{g} \cdot \mathrm{kg}^{-1} \cdot \mathrm{h}^{-1}\right)$ por vias venosa e peridural para analgesia pós-operatória de pacientes submetidos à intervenção cirúrgica intra-abdominal, foram observados os mesmos níveis de analgesia e incidência de efeitos colaterais, como náusea e sedação, nos dois grupos de estudo, apesar de os pacientes que receberam o opióide por via venosa apresentarem sedação mais intensa ${ }^{37}$. Este estudo mostrou que a analgesia e os efeitos adversos são resultantes da captação plasmática e redistribuição para os receptores opióides encefálicos e periféricos e não da ação medular.

Os opióides movem-se rapidamente do espaço peridural para o líquor e para a medula espinhal por difusão simples através das meninges ${ }^{33}$. Seu comportamento no espaço subaracnóideo é determinado pela solubilidade lipídica. Foi demonstrado que os opióides hidrofóbicos têm volume aparente de distribuição elevado e este é resultado de sua rápida saída do meio aquoso do líquor em direção aos meios mais hidrofóbicos, como a gordura peridural ${ }^{38}$.

Cerca de $60 \%$ da dose de sufentanil administrada no espaço subaracnóideo passam para o espaço peridural, ganhando o sistema circulatório ${ }^{38}$.

É provável que os opióides administrados pela via subaracnóidea possuam ação espinhal, mas eles também passam para o plasma onde se distribuem para o encéfalo, produzindo sedação e depressão respiratória ${ }^{33}$.

Os resultados mostraram que o sufentanil nas doses utilizadas nesta pesquisa, administrado pela via subaracnóidea ou peridural, associado aos anestésicos locais, determinou similaridade na duração do trabalho de parto após analgesia e no boletim de Apgar dos recém-nascidos. Com relação aos efeitos colaterais, apenas a sedação foi mais freqüente nas pacientes que receberam o opióide por via peridural.

\section{Side Effects of Subarachnoid and Epi- dural Sufentanil Associated with a Local Anesthetic in Patients Undergoing Labor Analgesia}

Isabel C. F. Salem, M.D.; Fernanda B. Fukushima, M.D.; Giane Nakamura, M.D.; Fábio Ferrari, TSA, M.D.; Laís C. Navarro, M.D.; Yara Marcondes Machado Castiglia, TSA, M.D.; Eliana Marisa Ganem, TSA, M.D.

\section{INTRODUCTION}

Pain represents one of the most important clinical signs of the beginning of labor and, once the diagnosis is made and the regularity of uterine contractions established, pain must be treated since it has several undesirable effects both in the mother and in the fetus.

Anxiety and pain during the phase of cervical dilation lead to maternal hyperventilation, increase in oxygen consumption and in circulating catecholamines, cortisol, and adrenocorticotrophic hormone ${ }^{1}$. Hyperventilation causes hypocarbia, uteroplancental vasoconstriction, and deviation of maternal hemoglobin dissociation curve to the left; these effects, associated with increased maternal oxygen consumption decrease oxygen delivery to the fetus. Besides uterine vasoconstriction ${ }^{1}$, excessive concentration of circulating catecholamines increases oxygen consumption ${ }^{1}$ and blood lactate ${ }^{1}$. Pain relief during labor decreases minute ventilation, oxygen consumption, concentration of circulating catecholamines, and blood lactate ${ }^{1}$. Studies conducted after epidural anesthesia for labor analgesia demonstrated improvement in placental perfusion and a reduction in the concentration of the substances released during periods of stress, such as cortisol and $\beta$-edorphins ${ }^{1}$.

Modern anesthetic techniques, associated with the development of new local anesthetics with decreased cardiovascular and neurological toxicity, and the addition of opioids to local anesthetics, are responsible for pain free labor with few maternal and fetal side effects ${ }^{1-3}$.

Local anesthetics (LA) blocks sodium channels in the membrane of axons in the spinal nerve roots and in the cells of the posterior and anterior horns of the spinal cord. Opioids are agonists of pre- and post-synaptic opioid receptors in the central nervous system and in other areas of the body ${ }^{4,5}$. These drugs, with distinct mechanisms of action, have synergistic action ${ }^{6}$.

Adding opioids, such as fentanyl and sufentanil, to LA decreases the latency, prolongs the duration, and improves the quality of epidural anesthesia ${ }^{1}$. This association also allows the anesthesiologist to use low concentrations of LA, decreasing the motor blockade and the risk of toxicity ${ }^{1}$.

Another technique often used for pain relief during labor is the combined spinal-epidural (spinal block and epidural 
anesthesia), with the association of bupivacaine and subarachnoid liposoluble opioids (fentanyl or sufentanil) ${ }^{7}$. The advantages of subarachnoid opioids include improvement in the quality of analgesia ${ }^{8}$, fast onset of action ${ }^{8}$, absence of motor blockade ${ }^{8}$, decreased maternal/fetal exposure to drugs, and the technique is easy to perform ${ }^{9}$. The main disadvantages of using opioids in the neuroaxis include pruritus, hypotension, temporary changes in fetal heart rate, and respiratory depression, whose incidence varies from $0.01 \%$ to $0.1 \%$ and is not significant in the absence of sedation ${ }^{10}$.

The incidence of side effects is decreased by reducing the dose of the subarachnoid opioid ${ }^{11}$. When $5 \mu \mathrm{g}$ of sufentanil was associated with subarachnoid bupivacaine in the technique of combined spinal-epidural, the quality of analgesia was preserved and the incidence of side effects was minimal ${ }^{12}$

The aim of this study was to compare the side effects of the association of subarachnoid sufentanil and bupivacaine with those caused by the association of epidural sufentanil and ropivacaine, in the doses used in the Anesthesiology Department, in pregnant women undergoing labor analgesia.

\section{METHODS}

After approval by the Ethics Committee on Clinical Research and signing of the informed consent, 60 pregnant women, between 15 and 42 years old, ASA physical status I and II, with full term pregnancies, healthy fetuses without fetal distress, who underwent labor analgesia participated in this study. Patients with current or past gastric complaints, those who used alcohol or drugs, patients with psychiatric disorders, and patients who received meperidine during labor were excluded. Patients were randomly divided in two groups that differed regarding the anesthetic technique used:

- Group 1 (combined spinal-epidural): 0.5\% bupivacaine $(2.5 \mathrm{mg})+$ subarachnoid sufentanil $(5 \mu \mathrm{g})$

- Group 2 (epidural): $0.2 \%$ ropivacaine $(14 \mathrm{mg})+$ epidural sufentanil $(10 \mu \mathrm{g})$

Complementary doses: epidural $0.2 \%$ ropivacaine $(12 \mathrm{mg}$ ) in both groups. Dosage for labor resolution: epidural $1 \%$ ropivacaine $(50 \mathrm{mg})$

After the indication of labor analgesia, which in the Hospital das Clínicas de Botucatu is done when the cervical dilation is equal to $6 \mathrm{~cm}$, Ringer's lactate ( 8 to $10 \mathrm{~mL} \cdot \mathrm{kg}^{-1}$ ) infusion was initiated and patients were monitored with an electrocardioscope in the $D_{\|}$derivation, sphygmomanometer, and pulse oxymeter.

In Group 1, an epidural puncture was performed with the patient in the sitting position, by the median approach, in the $\mathrm{L}_{3}-\mathrm{L}_{4}$ space with a Touhy $80 / 18$ needle and an epidural catheter was inserted, followed by a subarachnoid puncture in the $L_{2}-L_{3}$ space with a 25G Quincke needle and the administration of $5 \mu \mathrm{g}$ of sufentanil and $2.5 \mathrm{mg}$ of bupivacaine. In patients who needed further doses for analgesia, a test dose of a local anesthetic ( $1 \%$ lidocaine - $30 \mathrm{mg}$ and adrenaline $1: 200,000)$ was administered to confirm it was properly placed. It was followed by $6 \mathrm{~mL}(12 \mathrm{mg})$ of $0.2 \%$ ropivacaine. In Group 2, the epidural puncture was performed using the same technique as in Group 1 and, after the test dose, 10 $\mathrm{mL}(20 \mathrm{mg})$ of $0.2 \%$ ropivacaine and sufentanil $(10 \mu \mathrm{g})$ were administered. Whenever patients needed additional doses of anesthetic due to pain, the complementary dose of $0.2 \%$ ropivacaine, $6 \mathrm{~mL}$ (12 $\mathrm{mg})$, was administered.

For labor resolution, $5 \mathrm{~mL}$ of $1 \%$ ropivacaine were administered.

Determination of side effects was done in two moments: M1 - the period of labor after analgesia, and M2 - 24 hours after analgesia.

The period of time from analgesia until the patient left the delivery room was considered as the period of labor after analgesia, and the presence of hypotension (at least a $20 \%$ drop in blood pressure), bradycardia (heart rate below 60 beats per minutes), pruritus, sedation, nausea, vomiting, and respiratory depression was evaluated every 10 minutes.

The Apgar scores of the newborns were determined.

In case of hypotension, the patient was initially treated by moving the uterus to the left and increasing the rate of the IV infusion. If those measures were ineffective, ephedrine was administered. Pruritus would be treated with propofol (10 mg), and nausea with ondansetron (4 mg).

In the first 24 hours after analgesia, patients were oriented to request the presence of an anesthesiologist if they developed pruritus, nausea, and pain, when they would receive the medication established in the protocol.

In the postoperative period, 24 hours after analgesia (M2), patients were evaluated by another anesthesiologist who was present during the anesthesia to determine whether the patients experienced pain, nausea, vomiting, pruritus, sedation, and urinary retention. These events were evaluated during an interview, with no prior knowledge of which group the patient belonged to.

Sedation was evaluated by the sedation numeric scale that varies from 0 to 3 , where $0=$ patient awake, without clinical evidence of sedation; 1 = sleepy, responding to verbal commands; 2 = sleepy, not responding to verbal commands, but responding to light touch; and 3 = severe sedation, responding to painful stimuli. Pruritus was also evaluated using a numeric scale from 0 to 3 , where $0=$ absent; $1=$ light pruritus, without the need to scratch; 2 = moderate pruritus, with occasional scratching; and $3=$ severe pruritus, scratching constantly. Pain was evaluated by the verbal numeric scale, from 0 to 10 , where 0 is the absence of pain and ten stands for maximal pain. When pain severity was greater than 3 , oral cetoprophen $(50 \mathrm{mg}$ ) was administered according to the norms established by the Obstetric Department. Considering a $40 \%$ difference between the groups and the power of the test of $95 \%$, the size of the study population was calculated as a minimum of 22 patients per group. 
In the statistical analysis of the results, the test $t$ Student was used for quantitative variables with normal distribution and the Mann-Whitney test for those that were not. The Chisquare test was used for qualitative variables.

\section{RESULTS}

Both groups were homogenous regarding the anthropometric data, duration of labor after analgesia (Table I), and Apgar score of the newborns (Table II). Only two patients in Group 1 needed complementary doses of epidural anesthetic. In both cases, a single dose was administered 90 and 110 minutes after the initial dose. Five patients in Group 2 needed complementary doses of ropivacaine, also as a single dose for each patient, 100 to 195 minutes after the initial dose.

Of the 60 patients selected for the study, eight were excluded from the analysis of the results because they had cesarean sections. Of the 52 remaining patients, 28 were in Group 1, and 24 in Group 2.

Five patients in Group 1 and four in Group 2 had an episiotomy for relief forceps.

Of the 28 patients in Group 1, 19 presented one or more side effects during the study period; three of them in M1, eight in M1 and $\mathrm{M} 2$, and eight in M2. Pruritus was the most common symptom in $\mathrm{M} 1$, affecting eight patients (six mild, and two moderate), followed by hypotension and nausea, which affected four patients (Table III). The patients with hypotension were not the same that complained of nausea. Pruritus did not require any treatment. Hypotension was treated moving the uterus to the left and increasing the infusion of IV fluids. Nausea was transitory and did not require any treatment. In $\mathrm{M} 2$, eight patients complained of pruritus, followed by pain and sedation, which affected six patients (Table IV).

Table I - Anthropometric Data and Duration of M1 (Mean \pm SD)

\begin{tabular}{lccc}
\hline & Group 1 & Group 2 & Statistics \\
\hline Age (years) & $20.6 \pm 4.1$ & $22.6 \pm 6.6$ & $\mathrm{G} 1=\mathrm{G} 2$ \\
Weight $(\mathrm{kg})$ & $72.4 \pm 8.2$ & $72.1 \pm 10.4$ & $\mathrm{G} 1=\mathrm{G} 2$ \\
Height $(\mathrm{cm})$ & $162 \pm 6$ & $161 \pm 6$ & $\mathrm{G} 1=\mathrm{G} 2$ \\
Duration of M1 $(\mathrm{min})$ & $152 \pm 3$ & $175 \pm 14$ & $\mathrm{G} 1=\mathrm{G} 2$ \\
\hline
\end{tabular}

Table II - Apgar Scores of the Newborns. Results Expressed as the Median

\begin{tabular}{lccl}
\hline Moment & Group 1 & Group 2 & Statistics \\
\hline $1 \mathrm{~min}$ & $8[7.5-8.5]$ & $8[8-9]$ & $\mathrm{G} 1=\mathrm{G} 2$ \\
$5 \mathrm{~min}$ & $9[9-10]$ & $9[9-10]$ & $\mathrm{G} 1=\mathrm{G} 2$ \\
$10 \mathrm{~min}$ & $10[9-10]$ & $10[9-10]$ & $\mathrm{G} 1=\mathrm{G} 2$ \\
\hline
\end{tabular}

Table III — Side Effects Observed at M1

\begin{tabular}{lccc}
\hline & $\begin{array}{c}\text { Group 1 } \\
\mathrm{n}(\%)\end{array}$ & $\begin{array}{c}\text { Group } 2 \\
\mathrm{n}(\%)\end{array}$ & Statistics \\
\hline Hypotension & $4(14)$ & $1(4)$ & $\mathrm{G} 1=\mathrm{G} 2$ \\
Bradycardia & 0 & $1(4)$ & $\mathrm{G} 1=\mathrm{G} 2$ \\
Pruritus & $8(29)$ & $2(8)$ & $\mathrm{G} 1=\mathrm{G} 2$ \\
Nausea & $4(14)$ & 0 & $\mathrm{G} 1=\mathrm{G} 2$ \\
Vomiting & $2(7)$ & 0 & $\mathrm{G} 1=\mathrm{G} 2$ \\
Respiratory depression & 0 & 0 & $\mathrm{G} 1=\mathrm{G} 2$ \\
Sedation & $2(7)$ & $12(50)$ & $\mathrm{G} 1<\mathrm{G} 2$ * \\
\hline
\end{tabular}

${ }^{*} p<0.05$

Table IV - Adverse Effects Observed at M2. Results Expressed in Absolute Numbers ( $\mathrm{n}$ ) and Percentage (\%)

\begin{tabular}{lccc}
\hline & $\begin{array}{c}\text { Group } 1 \\
\mathrm{n}(\%)\end{array}$ & $\begin{array}{c}\text { Group } 2 \\
\mathrm{n}(\%)\end{array}$ & Statistics \\
\hline Pain & $7(25)$ & $3(12.5)$ & $\mathrm{G} 1=\mathrm{G} 2$ \\
Nausea & $3(11)$ & $3(12.5)$ & $\mathrm{G} 1=\mathrm{G} 2$ \\
Vomiting & 0 & 0 & $\mathrm{G} 1=\mathrm{G} 2$ \\
Pruritus & $9(32)$ & $4(17)$ & $\mathrm{G} 1=\mathrm{G} 2$ \\
Sedation & $6(21)$ & $5(21)$ & $\mathrm{G} 1=\mathrm{G} 2$ \\
Urinary retention & $4(14)$ & $1(4)$ & $\mathrm{G} 1=\mathrm{G} 2$ \\
\hline
\end{tabular}

Eighteen patients in Group 2 had one or more side effects during the evaluation period; eight in $M 1$, seven in $M 1$ and M2, and three in M2. Twelve patients complained of sedation in M1 (Table III) and five in M2, four of which had also complained of sedation in M1. Ten patients presented grade 1 sedation and three, grade 2 sedation. Pruritus was the second most common side effect, being classified as mild to moderate (two patients in M1 and four in M2) (Table IV), which did not require treatment. The incidence of sedation in $M 1$ in Group 2 was greater than in Group 1, which was statistically significant $(p=0.017)$.

\section{DISCUSSION}

The results showed that subarachnoid or epidural sufentanil, in the doses used in this study, associated with local anesthetics had a similar behavior regarding duration of analgesia and vitality of the newborns, evaluated by the Apgar score.

The incidence of adverse effects, such as pruritus, bradycardia, hypotension, nausea, vomiting, respiratory depression, and urinary retention was also similar in both groups. Only the incidence of sedation in M1 was greater in patients who received epidural sufentanil, which was statistically significant. 


\section{SIDE EFFECTS OF SUBARACHNOID AND EPIDURAL SUFENTANIL ASSOCIATED WITH}

A LOCAL ANESTHETIC IN PATIENTS UNDERGOING LABOR ANALGESIA

The administration of sufentanil in the neuroaxis is associated with several side effects. Although some of them reflect their action in the spinal cord, others are caused by the cephalic migration and supraspinal effects ${ }^{13}$.

Pruritus is caused by spinal and supraspinal actions ${ }^{11}$. It is the most frequent side effect ${ }^{14}$. Although there were no statistically significant differences, pruritus was the main complaint of $32 \%$ of the patients who underwent combined spinal-epidural and $17 \%$ of those who underwent epidural anesthesia.

These results are in agreement with those in the literature, in which the incidence of pruritus is greater after the administration of subarachnoid opioid ${ }^{15}$. The incidence after the subarachnoid administration of sufentanil varies from $25 \%$ to $90 \%{ }^{11-13,16-18}$.

In a study with pregnant women who underwent analgesia by the combined spinal-epidural technique with $5 \mu \mathrm{g}$ or 10 $\mu \mathrm{g}$ of sufentanil, it was observed that reducing the dose of the opioid did not change the severity of the pruritus ${ }^{13}$.

Some authors ${ }^{11}$ observed that the incidence of hypotension and sedation was reduced using $5 \mu \mathrm{g}$, compared with $10 \mu \mathrm{g}$, of sufentanil administered in the subarachnoid space. Similar results were observed with doses of sufentanil ranging from 2.5 to $10 \mu g^{18}$.

Similar to what happens with the analgesic effect, there seem to be a threshold above which sufentanil produces pruritus regardless of the dose administered ${ }^{13}$.

It is still unclear whether pruritus is produced by the cephalic migration of the drug in the spinal fluid and subsequent interaction with the nucleus of the trigeminal nerve in the medulla ${ }^{19}$, or if type 3 serotonin receptors are involved, since they are abundant in the dorsal horn of the spinal cord ${ }^{19}$.

Fourteen percent of the patients who underwent combined spinal-epidural experienced hypotension, which is similar to the incidence reported by other authors ${ }^{7,11,12}$.

Hypotension caused by subarachnoid and epidural sufentanil depends on the dose of the opioid, as well as on the association with the local anesthetic and its dose.

Several studies with pregnant women demonstrated that the incidence of hypotension with the association of bupivacaine $(2.5 \mathrm{mg})$ and subarachnoid sufentanil $(10 \mu \mathrm{g})$ varied from $11 \%{ }^{7}$ to $43 \%{ }^{11}$. Adding $7.5 \mu \mathrm{g}$ of sufentanil to bupivacaine $(2.0 \mathrm{mg}$ ) caused hypotension in $15 \%$ of the patients, while the incidence decreased between $5 \%$ to $9 \%$ adding $5 \mu \mathrm{g}$ to bupivacaine $(1.25 \mathrm{mg}){ }^{11,12}$.

Using a non-invasive method of thoracic bioimpedance to monitor the central hemodynamics in pregnant women undergoing combined spinal-epidural, which included the subarachnoid administration of sufentanil $(10 \mu \mathrm{g}), 10 \%$ of the patients developed hypotension without changes in central cardiovascular parameters ${ }^{20}$.

It is well established that hypotension secondary to the subarachnoid administration of sufentanil for labor analgesia is secondary to the immediate and profound analgesia caused by the technique, leading to the immediate decrease in the concentration of circulating catecholamines ${ }^{21}$.

Fourteen percent of the patients in Group 1 complained of nausea in $\mathrm{M} 1$ and $12 \%$ of the patients in both groups presented this complaint in M2.

The incidence of nausea in pregnant women after the administration of subarachnoid sufentanil with doses ranging from $1 \mu \mathrm{g}$ to $10 \mu \mathrm{g}$ varied from $0 \%$ to $35 \%{ }^{7,11,12,18,21}$.

When sufentanil $(10 \mu \mathrm{g})$ was associated with $0.2 \%$ ropivacaine and administered in the epidural space for labor analgesia, $20 \%$ of the patients complained of nausea ${ }^{23}$.

In the postoperative analgesia of abdominal surgeries, the continuous infusion of epidural sufentanil led to vomiting in $33 \%$ of the patients ${ }^{24}$.

When associated with bupivacaine in the treatment of post cesarean section pain, sufentanil caused nausea in $12 \%$ of the patients ${ }^{25}$.

However, some studies did not demonstrated vomiting in patients who received up to $30 \mu \mathrm{g}$ of epidural sufentanil for the treatment of post cesarean section pain ${ }^{25}$.

Opioids can cause nausea and vomiting because they stimulate the chemoreceptor area of the trigger zone on the floor of the fourth ventricle, reflecting its characteristic of partial agonist of dopaminergic receptors in the chemoreceptor zone. The blood-brain barrier is more permeable in this area than in other regions of the brain ${ }^{26}$.

Fifty percent of the patients who received epidural sufentanil presented sedation. These results are higher than those found in the literature.

Vertommen et al. ${ }^{27}$ observed that only $8 \%$ of the patients receiving epidural bupivacaine $(12.5 \mathrm{mg})$ and sufentanil (10 $\mu \mathrm{g}$ ) for labor analgesia presented mild somnolence.

Somnolence was also reported by $30 \%$ of the patients who underwent cesarean sections and received epidural sufentanil $(30 \mu \mathrm{g}){ }^{28}$.

Other authors, who administered a continuous infusion of $0.1 \%$ ropivacaine $(10 \mathrm{~mL})$ associated with sufentanil $\left(2 \mu \mathrm{g} \cdot \mathrm{mL}^{-1}\right)$ for labor analgesia, observed somnolence in $25 \%$ of the patients ${ }^{29}$. It was observed that the sedation caused by sufentanil is more pronounced than the one caused by fentanyl ${ }^{24}$. In postoperative analgesia of abdominal surgeries, the continuous infusion of epidural sufentanil $\left(5 \mu \mathrm{g} \cdot \mathrm{h}^{-1}\right)$ promoted light sedation in the first four hours of infusion ${ }^{24}$. In the present study, the greater incidence of sedation was observed in M1 and it was also premature in the first three hours after the administration of sufentanil.

The incidence of sedation at M1 in patients who underwent combined spinal-epidural was $7 \%$.

The incidence of sedation in pregnant women, who received combined spinal-epidural and in those who received only sufentanil or associated with a local anesthetic, varies tremendously.

Mild sedation is reported in $100 \%$ of pregnant women who received $10 \mu \mathrm{g}$ of sufentanil in the subarachnoid space ${ }^{25}$. Another study showed similar results with this dose ${ }^{30}$. 
Other authors reported that doses of sufentanil from $1 \mu \mathrm{g}$ to $10 \mu \mathrm{g}$ administered in the subarachnoid space for labor analgesia caused somnolence in $30 \%$ of the patients, regardless of the dose administered. In another study, the subarachnoid administration of $8 \mu \mathrm{g}$ of sufentanil caused sedation in $58 \%$ of the patients ${ }^{31}$.

When associated with bupivacaine, sufentanil $(7.5 \mu \mathrm{g})$ caused mild sedation in $12.5 \%$ of the patients ${ }^{22}$. After the administration of $5 \mu \mathrm{g}, 5 \%$ of the patients presented this symptom ${ }^{11}$.

Although sedation is a common side effect of opioids, the mechanism and characteristics of this phenomenon are not completely understood. In a study on the probable mechanisms of the interaction between opioids and benzodiazepines, the hypothesis of the co-localization of gamma-aminobutyric acid (GABA) receptors and opioid receptors in the central nervous system was raised, with possible cross activity and common pathways of intracellular transduction ${ }^{32}$.

To exert their effects, opioids administered epidurally have to cross the dura mater and the arachnoid, diffuse in the cerebrospinal fluid, cross the pia mater, reach the surface of the spinal cord, and diffuse through the white matter and the gray matter to reach the opioid receptors in the dorsal horn ${ }^{33}$.

In the epidural space, opioids can be captured by the epidural fat, absorbed into the systemic circulation, or diffuse through the dura mater into the cerebrospinal fluid ${ }^{34}$. Since the epidural space has an extensive venous plexus, vascular absorption is intense. The epidural administration of fentanyl and sufentanil produces blood concentrations similar to the venous administration of equivalent doses ${ }^{34}$.

Some authors demonstrated that fentanyl exerts spinal actions when administered in the epidural space ${ }^{35}$, and several studies demonstrated that fentanyl ${ }^{36}$ and sufentanil ${ }^{37}$, administered in the epidural space, produced the same degree of analgesia and the same side effects as the intravenous administration. These studies emphasize the importance of the systemic absorption and subsequent cephalic distribution in the quality of analgesia and severity of side effects when lipophilic opioids are used ${ }^{33}$.

A prospective, random, double blind study using continuous intravenous and epidural infusion of sufentanil $\left(0.2 \mu \mathrm{g} \cdot \mathrm{kg}^{-1} \cdot \mathrm{h}^{-1}\right)$ for postoperative analgesia of patients who underwent abdominal surgeries, observed the same levels of analgesia and incidence of side effects, such as nausea and sedation, in both study groups, although patients who received intravenous opioids had more severe sedation ${ }^{37}$. This study showed that analgesia and side effects result from the absorption of the plasma and the redistribution to central and peripheral opioid receptors and not from the action in the spinal cord.

Opioids move rapidly from the epidural space to the cerebrospinal fluid and to the spinal cord by simple diffusion through the meninges ${ }^{33}$. Their behavior in the subarachnoid space is determined by their lipid solubility. It has been shown that hydrophobic opioids have a high apparent volume of distribution resulting from the rapid exit from the aqueous medium of the cerebrospinal fluid to more hydrophobic spaces, such as the epidural fat ${ }^{38}$.

Approximately $60 \%$ of the dose of sufentanil administered in the subarachnoid space traverse to the epidural space and access the circulatory system ${ }^{38}$.

Opioids administered in the subarachnoid space probably have a spinal action, but they also diffuse to the plasma, being distributed to the brain, producing sedation and respiratory depression ${ }^{33}$.

The results demonstrated that sufentanil, in the doses used in this study, administered in the subarachnoid or epidural space, associated with local anesthetics, had similar effects in the duration of labor after analgesia and in Apgar scores of the newborns. Regarding side effects, only sedation was more frequent in patients who received epidural opioids.

\section{REFERÊNCIAS - REFERENCES}

01. Thomas - Anesthesia for labor and delivery. Probl Anesth, 1999;11:307-323.

02. Castro LFL, Serafim MM, Côrtes CAF et al. - Avaliação do estado ácido-base materno com o uso de sufentanil por via subaracnóidea em diferentes doses para cesarianas e suas repercussões sobre os recém-nascidos. Rev Bras Anestesiol, 2003;1:17-24.

03. Nakamura G, Castiglia YMM, Nascimento Jr P et al. - Bupivacaína, ropivacaína e levobupivacaína em analgesia e anestesia de parto. Repercussões materno-fetais. Rev Bras Anestesiol, 2000;50:105-111.

04. Pleuvry BJ - Opioid receptors and their relevance to anaesthesia. Br J Anaesth, 1993; 72:119-126.

05. Stein $\mathrm{C}-$ The control of pain in peripheral tissue by opioids. $\mathrm{N}$ Engl J Med, 1995;332:1685-1690.

06. Hepner D, Dalta $S$ - Labor analgesia pratices for the new millennium. Sem Anesth Periop Med Pain, 2000;19:35-45.

07. Campbell DC, Camann WR, Datta $S$ et al. - The addiction of bupivacaine to intrathecal sufentanil for labor analgesia. Anesth Analg, 1995;81:305-309.

08. D'Angelo R, Anderson MT, Philip J et al. - Intrathecal sufentanil compared to bupivacaine for labor analgesia. Anesthesiology, 1994;80:1209-1215.

09. Riley ET, Ross BK - Epidural and Spinal Analgesia/Anesthesia. Section II: Opioid Techniques, em: Chestnut DH - Obstetric Anesthesia: Principles and Practice. $2^{\text {nd }}$ Ed, St Louis, Mosby, 1999;394-400.

10. Eisenach JC - Combined spinal-epidural analgesia in obstetrics. Anesthesiology, 1999; 91:299-302.

11. Sia AT, Chong JL, Chiu JW - Combination of intrathecal sufentanil $10 \mu \mathrm{g}$ plus bupivacaine $2,5 \mathrm{mg}$ for labor analgesia: is half the dose enough? Anesth Analg, 1999;88:362-366.

12. Cheng CJ, Sia AT, Lim EH et al. - Either sufentanil or fentanyl, in addition to intrathecal bupivacaine, provide satistactory early labour analgesia. Can J Anaesth, 2001;48:570-574.

13. Norris MC, Fogel ST, Holtmann B - Intrathecal sufentanil ( 5 vs $10 \mu \mathrm{g})$ for labor analgesia: efficacy and side effects. Reg Anesth Pain Med, 1998;23:252-257.

14. Honet JE, Arkoosh VA, Norris MC et al. - Comparison among intrathecal fentanyl, meperidine and sufentanil for labour analgesia. Anesth Analg, 1992;75:734-739. 


\section{SIDE EFFECTS OF SUBARACHNOID AND EPIDURAL SUFENTANIL ASSOCIATED WITH A LOCAL ANESTHETIC IN PATIENTS UNDERGOING LABOR ANALGESIA}

15. Cousins MJ, Mather LR - Intrathecal and epidural administration of opioids. Anesthesiology, 1984; 61:276-310.

16. Yamaguchi ET, Carvalho JCA, Fonseca US et al. - Sufentanil subaracnóideo associado à bupivacaína hiperbárica para analgesia de parto: é possível reduzir a dose de opióide? Rev Bras Anestesiol, 2004:54:145-152.

17. Fournier R, Weber A, Gamulin Z - Intrathecal sufentanil is more potent than intravenous for postoperative analgesia after totalhip replacement. Reg Anesth Pain Med, 2005;30:249-254.

18. Camann WR, Abouleish A, Eisenach JC et al. - Intrathecal sufentanil and epidural bupivacaine for labor analgesia: doseresponse of individual agents and combination. Reg Anesth Pain Med, 1998;23:457-462.

19. Gürkan $\mathrm{Y}$, Toker $\mathrm{K}$ - Prophylatic ondansetron reduces pruritus. Anesth Analg, 2002;95:1763-1766.

20. Pham LH, Camann WR, Smith MP et al. - Hemodynamic effects of intrathecal sufentanil compared with epidural bupivacaine in laboring parturients. J Clin Anesth, 1996;8:497-501.

21. Riley ET, Ratner EF, Cohen SE - Intrathecal sufentanil for labor analgesia: do sensory changes predict better analgesia and greater hypotension? Anesth Analg, 1997:84:346-251.

22. Eriksson SL, Blomberg I, Olofsson C - Single-shot intrathecal sufentanil with bupivacaine in late labour-analgesic quality and obstetric outcome. Eur J Obst Gynecol Reprod Biol, 2003; 110:131-135.

23. Debon R, Bernard A, Duflo $F$ et al. - The analgesic effect of sufentanil combined with ropivacaine $0.2 \%$ for labor analgesia: a comparison of three sufentanil doses. Anesth Analg, 2001; 92:180-183.

24. Geller E, Chrubasik J, Graf R et al. - A randomized double-blind comparison of epidural sufentanil versus intravenous sufentanil or epidural fentanyl analgesia after major abdominal surgery. Anesth Analg, 1993;76:1243-1250.

25. Cohen S, Amar D, Pantuck CB et al. - Postcesarean delivery epidural patient-controlled analgesia. Fentanyl or sufentanil? Anesthesiology, 1993;78:486-491.

26. Malan TP - Opioid pharmacology: new insights and clinical relevance. ASA Refresher Course, 2000; 28:109-119.

27. Vertommen JD, Vandermeulen E, Aken HV et al. - The effects of the addition of sufentanil to $0,125 \%$ bupivacaine in late quality of analgesic during labor and on the incidence on instrumental deliveries. Anesthesiology, 1991;74:809-814.

28. Cohen Se, Tan S, White PF - Sufentanil analgesia following cesarean section: epidural versus intravenous administration. Anesthesiology, 1988;66:129-134.

29. Lee BB, Kee WDN, Lau WM et al. - Epidural infusions for labor analgesia: a comparison of $0.2 \%$ ropivacaine, $0.1 \%$ ropivacaine, and $0,1 \%$ ropivacaine with fentanyl. Reg Anesth Pain Med, 2002;27:31-36

30. Chaves IMM, Machado GP, Almeida JR et al. - Estudo comparativo do sufentanil por via peridural, subaracnóidea e venosa em cesarianas. Rev Bras Anestesiol, 1999;49:332-335.

31. Nelson KE, Rauch $T$, Terebuh $V$ et al. - A comparison of intrathecal fentanyl and sufentanil for labor analgesia. Anesthesiology, 2002;96:1070-1073.

32. Megarbane B, Gueye P, Baud F - Interactions between benzodiazepines and opioids. Ann Med Interne, 2003;154:s64-s72.

33. Bernards $\mathrm{CM}-$ Recent insights into the pharmacokinetics of spinal opioids and relevance to opioid selection. Curr Opin Anaesthesiol, 2004;17:441-447.

34. Chaney MA - Side effects of intrathecal and epidural opioids. Can J Anaesth, 1995;42:891-903.

35. D'Angelo R, Gerancher JC, Eisenach JC et al. - Epidural fentanyl produces labor analgesia by a spinal mechanism. Anesthesiology, 1998;88:1519-1525.
36. Ellis DJ, Millar WL, Reisner LS - A randomized double-blind comparison of epidural versus intravenous fentanyl infusion for analgesia after cesarean section. Anesthesiology, 1990; 72:981-986

37. Miguel R, Barlow I, Morrell M et al. - A prospective, randomized, double-blind comparison of epidural and intravenous sufentanil infusions. Anesthesiology, 1994;81:346-352.

38. Ummenhofer WC, Arends RH, Shen DD et al. - Comparative spinal distribution and clearance kinetics of intrathecally administered morphine, fentanyl, alfentanil, and sufentanil. Anesthesiology, 2000;92:739-753.

\section{RESUMEN}

Salem ICF, Fukushima FB, Nakamura G, Ferrari F, Navarro LC, Castiglia YMM, Ganem EM - Efectos Adversos del Sufentanil Asociado al Anestésico Local por las Vías Subaracnoidea y Peridural en Pacientes Sometidas a la Analgesia de Parto.

JUSTIFICATIVA Y OBJETIVOS: La asociación del opioide con el anestésico local mejora la calidad de la analgesia de parto y reduce el riesgo de toxicidad sistémica por el anestésico local. Los opioides, sin embargo, pueden determinar efectos colaterales. El objetivo de esta investigación fue comparar los efectos adversos determinados por el sufentanil, administrado por vía subaracnoidea, asociado a la bupivacaína, con aquel determinado por el sufentanil por vía peridural, asociado a la ropivacaína, en las dosis utilizadas en el Servicio de Anestesia, en embarazadas sometidas a la analgesia de parto.

MÉTODO: Participaron del estudio 60 pacientes, estado físico ASA I y II, con edad entre los 15 y los 42 años, con embarazo en tiempo y fetos saludables, sometidas a la analgesia de parto. Se distribuyeron aleatoriamente en de los grupos: G1 - Doble bloqueo bupivacaína a $0,5 \%(2,5 \mathrm{mg})$ y sufentanil $(5 \mu \mathrm{g})$ por vía subaracnoidea, G2 - Peridural - ropivacaína a 0,2\% (20 mg) y sufentanil $(10 \mu g)$ por vía peridural. Para dosis complementarias fue administrada ropivacaína a 0,2\% (12 $\mathrm{mg})$ y para resolución del parto, ropivacaína a $1 \%(50 \mathrm{mg})$. Las pacientes se evaluaron después de la analgesia (M1) con relación a la hipotensión arterial, bradicardia materna, prurito, náusea, vómito, depresión respiratoria y sedación. En el postoperatorio (M2), en cuanto a la presencia de náusea, vómito, prurito, sedación, retención urinaria y dolor. Los recién nacidos se evaluaron por el índice de Apgar. Para análisis estadístico, se utilizaron la prueba $t$ de Student, Mann-Whitney y Qui-cuadrado.

RESULTADOS: Los grupos fueron similares con relación a la edad, al peso, a la altura, a la duración del período de trabajo de parto después de la analgesia, al Apgar de los recién nacidos, a la existencia de hipotensión arterial, bradicardia, náusea, vómito, prurito y retención urinaria. La sedación fue más frecuente en las pacientes de G2, en M1 (50\%) con diferencia estadística significativa.

CONCLUSIONES: El sufentanil en las dosis utilizadas, administrado por vía subaracnoidea o peridural, asociado a los anestésicos locales, determinó similitud en la duración del trabajo de parto después de la analgesia y en el Apgar de los recién nacidos. La sedación fue el efecto adverso más frecuente en las pacientes que recibieron el opioide por vía peridural. 